\title{
Des peptides à activité anti-thrombotique dans les protéines du lait : un lien entre thérapeutique et diététique
}

Il est admis que les protéines du lait et spécialement les caséines ont un rôle nutritionnel : c'est la principale source d'acides aminés pour les jeunes mammiferes pendant la période de lactation. Mais à côté de ce rôle, elles peuvent être la source de peptides actifs, potentiellement libérés au cours de la digestion. De nombreuses activités ont ainsi été reconnues dans des séquences peptidiques extraites des protéines du lait : activités opiacées, anti-hypertensives, immunomodulatrices [1].

Au cours de la phase primaire de la coagulation du lait, la caséine $\kappa$ est scindée spécifiquement par la chymosine par un mécanisme analogue au clivage du fibrinogène par la thrombine; il existe des analogies de séquence entre la chaîne $\gamma$ de la molécule du fibrinogène et la caséine $\kappa$ de vache [2].

Deux séquences différentes de la molécule de fibrinogène sont impliquées dans la liaison au complexe glycoprotéinique IIb/IIIa de la membrane plaquettaire: une séquence dodécapeptidique (les résidus 400 à 411) de la région carboxy-terminale de la chaîne $\gamma$ qui se fixe au niveau d'une séquence peptidique bien définie sur la glycoprotéine IIb de la plaquette [3] et une séquence tétrapeptidique de type $\mathrm{RGD} / \mathrm{x}^{*}$ de la chaîne $\alpha$ : il existe une séquence RGDF* (résidus 95 à 98) et une séquence RGDS* (résidus 572 à 575) sur la chaîne $\alpha$ mais il semble que ce soit la séquence RGDF qui soit principalement impliquée [4].

La première étape de la coagulation du lait est la scission de la molécule de caséine $\kappa$, qui conduit alors à la formation du caséino-glycopeptide (résidus 106 à 169). Précédemment, nous avons montré qu'à l'extrémité $\mathrm{N}$-terminale du caséino-glycopeptide de vache, il existe une séquence undécapeptidique (résidus 106 à 116) qui présente non seulement une homologie de séquence avec le dodécapeptide de la chaîne $\gamma$ du fibrinogène mais surtout une homologie fonctionnelle : l'undécapeptide du caséino-glycopeptide est plus puissamment anti-agrégant sur les plaquettes humaines que ne l'est le dodécapeptide du fibrinogène lui-même [5]. L'activité inhibitrice de l'undécapeptide peut être maintenue en réduisant la séquence à celle d'un pentapeptide, le KNQDK*. Dans les premiers tests pratiqués in vivo chez les petits rongeurs (rat et cobaye), dans un modèle de thrombose expérimentale induite par lésion endothéliale spécifique grâce à un rayon laser pulsé appliqué sur les artérioles du mésentère, certaines de ces séquences peptidiques, issues de la caséine $\kappa$, ont une activité anti-thrombotique. Les petits peptides ont cependant, comme il est prévisible, une courte durée de vie. Il paraît hautement probable que le site d'action sur la plaquette humaine des peptides provenant de l'extrémité $\mathrm{N}$-terminale du caséino-glycopeptide soit voisin de celui du dodécapeptide de la partie C-terminale de la chaîne $\gamma$ du fibrinogène.

Un effort important porte depuis plusieurs années sur l'étude de l'activité anti-thrombotique des séquences $\mathrm{RGD} / \mathrm{x}$ du fibrinogène mais, comme la séquence RGDS induit le détachement des cellules endothéliales in vitro [6], une toxicité générale peut être redoutée si ces séquences sont injectées in vivo. Une séquence n'ayant pas les effets délétères potentiels d'un RGD/x a été recherchée dans les protéines du lait. L'étude systématique des homologies de séquence a permis de découvrir une séquence qui s'apparente au RGD/x. Il s'agit de la séquence KRDS présente dans un tour $\beta$ de la lactotransferrine humaine (en position 39-42). Ce peptide a été synthétisé, ainsi que certains peptides analogues. Le KRDS est un peptide anti-thrombotique, mais il ne semble pas agir par le même mécanisme que le RGDS. In vitro, le KRDS est un inhibiteur de l'agrégation plaquettaire dépendante de l'ADP $\left(\mathrm{IC}_{50} 350 \mu \mathrm{M}\right)$ et de la fixation du fibrinogène aux plaquettes, mais de façon moins puissante que le $\operatorname{RGDS}\left(\mathrm{IC}_{50} 75 \mu \mathrm{M}\right)$. Le KRDS exerce également une action inhibitrice sur la fixation d'un anticorps monoclonal (P2) à activité anti-glycoprotéine IIb/IIIa sur des mégacaryocytes à un stade tardif de maturation [7]. En revanche, et contrairement au RGDS, il ne s'oppose pas à la fixation d'un autre anticorps monoclonal (PAC1) dirigé contre un épitope de configuration d'activation du groupe glycoprotéinique de membrane plaquettaire GP IIb/IIIa [8]. Contrairement au RGDS, il est inhibiteur de la sécrétion induite par la thrombine du contenu des granules de stockage des plaquettes humaines (55\%) pour une concentration de KRDS de $750 \mu \mathrm{M}$ ) et des plaquettes animales par un mécanisme qui est indépendant de la phosphorylation des protéines P20 et P43 [8]. De plus, cette inhibition de sécrétion (45\%) s'exerce sur des plaquettes de malades atteints de thrombasthénie de Glanzmann de type 1 (déficitaires en GP IIb/IIIa) excluant donc que cet effet inhibiteur passe par une action sur ce complexe glycoprotéinique. In vivo chez l'animal et ex vivo sur des plaquettes humaines et animales, le KRDS est inhibiteur de synthèse du thromboxane A2 (Wu et al., soumis pour publication). In vivo, le KRDS est anti-thrombotique dans trois modèles différents de thrombose expérimentale chez quatre espèces animales différentes. Nous avons été

* $R=$ arginine $G=$ glycine; $D=$ acide asparlique; $S=$ sérine; $F=$ phénylalanine; $K=$ lysine; $N=$ asparagine; $Q=$ glutamine; $V=$ valine. 
heureusement surpris de constater que cette action anti-thrombotique s'exerce pendant un temps plus long qu'attendu pour un tétrapeptide injecté, en tout cas plus long que celui observé pour le RGDV* dans un modèle simien [9]. Confirmant la différence de mode d'action entre KRDS et RGDS, nous avons pu montrer in vivo que les deux peptides ont une activité antithrombotique synergique dans le modèle de thrombose expérimentale induite par laser sur les artérioles du mésentère des petits rongeurs [10].

Actuellement, les travaux se poursuivent pour étudier l'effet de ces peptides du lait et d'autres, dans plusieurs fonctions hémostatiques : cofacteur de l'agrégation plaquettaire et dans la structuration de la fibrine si importante dans la thrombogenèse et la thrombolyse. Les quelques similitudes des exons codant pour le fibrinogène humain et la caséine $\kappa$ bovine représentent un abord moléculaire plein de promesse [11].

Dès lors que des peptides, originaires des protéines du lait, peuvent avoir une action préventive dans une pathologie aussi ubiquitaire que la thrombose, des perspectives nouvelles s'ouvrent dans les domaines de la nutrition, de la diététique ainsi que des possibilités de thérapeutiques préventives de masse [12]

\section{Jacques P. Caen}

Claire Bal dit Sollier

Élizabeth Mazoyer

Ludovic Drouet

Institut des vaisseaux et du sang, Hôpital Lariboisière, 8 rue Guy-Patin, 75010 Paris, France.

Pierre Jolles

Anne-Marie Fiat

Cnrs UA 1188, Laboratoire des protéines, Université Paris V, Paris, France.

\section{RÉFÉRENCES}

1. Fiat AM, Jolles P. Caseins of various origins and biologically active casein peptides and olisaccharides: structural and physiological aspects. Mol Cell Biochem 1989 ; 87: 5-30

2. Jolles $\mathrm{P}$, Loucheux-Lef ebvre MH, Henschen A. Structural relatedncss of k-casein and fibrinogen $\gamma$-chain. $J$ Mol Evol 1978 ; 11: $271-8$

3. D'Souza SE, Ginsberg MH, Matsueda GR, Plow EF. Discrete sequence in a platelet integrin is involved in ligand recognition. Nalure 1991；350 : 66-8

4. Andrieux A, Hudry-Clergeon G, Ryckwaert JJ, et al. Amino-acid sequences in fibrinogen mediating its interaction with its platelet receptor, GP IIb-IIIa. J Biol Chem 1989 ; 264: 9258-65.
5. Jolles P, Levy-Toledano S, Fiat AM, et al. Analogy between fibrinogen and casein effect of an undecapeptide isolated from $\kappa$ cascin on platelet function. Eur $J$ Biochem $1986 ; 158$ : 379-82

6. Bourdon MA, Ruoslahti E. Tenascin mediates cell attachment through an RGDdependent receptor. J Cell Biol $1989 ; 108$ : 1149-55.

7. Raha S, Dosquet C, Abgrall JF, Jolles P, Fiat AM, Caen JP. KRDS, a tetrapeptide derived from lactotransferrin inhibits binding of monoclonal antibody against glycoprotein IIb-IIIa on ADP stimulated platelets and megakaryocytes. Blood 1988 ; 72 : $172-8$

8. Mazoyer E, Levy-Toledano S, Rendu F, et al. KRDS, a new peptide derived from human lactotransferrin, inhibits platelet aggregation and release reaction. Eur J Biochem $1990 ; 194: 43-9$

9. Cadroy Y, Houghten RA, Hanson ST RGDV selectively inhibits platelet-dependent thrombus formation in vivo. I Clin Invest 1989 ; 84 : 939-44.

10 Drouet LO, Bal dit Sollier C, Cisse MT, et al. The anti-thrombotic effects of KRDS, a lactotransferrin peptide compared with RGDS. Nouv Rev Fr Hematol 1990 ; 32 : 59-62.

11. Alexander LJ, Stewart AF, Mac Kinlay $\mathrm{AG}$, el al. Isolation and characterisation of the bovine $\kappa$ casein genc. Eur $J$ Biochem 1988 ; 178 : 395-401.

12. Epidemiological studies of cardiovascular diseases. In : Progress Report Milk. Gwent : Lexprint, Risca, 1991: 35.

\section{TIRÉS A PART}

\section{J. Caen.}

\section{FLASH}

\section{UN GRAND PAS EN BIOLOGIE DE LA REPRODUCTION : SRY EST BIEN LE GĖNE DU DÉTERMINISME DU SEXE TDF}

Depuis maintenant vingt ans un grand nombre d'équipes a cherché à isoler le gène clé responsable du déterminisme primaire du sexe, qui donc va décider si un embryon doit devenir mâle. II y a maintenant plus de quarante ans, Alfred Jost a clairement montré que le gène du déterminisme du sexe devait être le gène de la différenciation des gonades en testicules chez le mâle ; ce gène clé a été dénommé TDF pour testis determining factor. C'est ce gène TDF qui vient d'être isolé et dont la fonction clé dans le choix du sexe vient d'être démontrée dans la revue Nature du 9 mai 1991 par le groupe du Dr R. Lovell-Badge. En 1990, et en collaboration avec notre laboratoire, $P$. Goodfellow et al. caractérisaient une séquence dénommée SRY et postulaient son rôle dans le déterminisme du sexe, car localisé sur $Y p$, conservé dans l'évolution et ayant une phase ouverte de lecture codant pour une protéine possédant un motif permettant la liaison à l'ADN. Le quatrième argument fut l'étude des femmes $X Y$ avec dysgénésie gonadique pure : il existait dans deux cas une mutation de novo dans $S R Y$.

La preuve définitive que SRY est bien TDF vient d'être apportée grâce à une expérience de transgénèse dans des souris femelles $X X$ d'un fragment génomique de $14 \mathrm{~kb}$ contenant l'homologue murin de SRY : Sry. Plus de trois cents embryons ont été microinjectés avec ce petit fragment d'ADN : quatre souris de caryotype $X X$ ont été obtenues ayant un phénotype mâle apparent tout à fait normal! Deux de ces souris ont été étudiées au stade 14 jours de développement embryonnaire et deux autres au stade adulte. Ces souris, porteurs de la séquence Sry, bien que de caryotype XX, ont des organes génitaux externes et internes mâles ; leurs gonades sont des testicules. Ce résultat démontre bien que Sry a joué le rôle que l'on attend du gène TDF : Induire les gonades embryonnaires en gonades mâles avec: al une organogenèse normale des cellules de Sertoli qui synthétisent l'hormone anti-Müllerienne, elle-même responsable de la régression des canaux de Müller qui auraient dû donner chez ces quatre souris $X X$ un utérus et des trompes; bl la présence de cellules de Leydig productrices des hormones androgènes et permettant le développement sans ambiguïté des organes génitaux externes mâles chez ces souris au génotype femelle.

Cependant, comme attendu, les deux souris $X X$ adultes sont stériles : en effet, Sry ou maintenant TDF, n'est, en revanche, pas le gène de la spermatogenèse qui, lui, est porté par le grand bras du chromosome $Y$ chez l'homme et qui, pour l'instant, n'a pas encore été isolé. Fait intéressant et plutôt surprenant, la transgénèse du gène SRY humain chez les souris XX n'a, quant à elle, aucun effet sur le développement normal des gonades en ovaires chez ces mêmes souris. Ce résultat semble suggérer que le produit de SRY interagit d'une manière spécifique d'espèce avec d'autres protéines ou d'autres gènes. Ce sont ces autres gènes de la cascade et leur fonction qu'il nous reste à découvrir maintenant.

Marc Fellous 